\title{
Optical Detection of Distal Lung Enzyme Activity in Human Inflammatory Lung Disease
}

\author{
Alicia Megia-Fernandez $\mathbb{D}^{1},{ }^{1}$ Adam Marshall $\left(\mathbb{D},{ }^{2}\right.$ Ahsan R. Akram ${ }^{(D)},{ }^{2}$ Bethany Mills ${ }^{D},{ }^{2}$ \\ Sunay V. Chankeshwara ${ }^{(D,}{ }^{1}$ Emma Scholefield ${ }^{D},{ }^{2}$ Amy Miele, ${ }^{2}$ Bruce C. McGorum ${ }^{(D)}{ }^{3}$ \\ Chesney Michaels, ${ }^{2}$ Nathan Knighton $\mathbb{D}^{4}{ }^{4}$ Tom Vercauteren ${ }^{(D)}{ }^{5}$ Francois Lacombe, ${ }^{6}$ \\ Veronique Dentan, ${ }^{6}$ Annya M. Bruce, ${ }^{2}$ Joanne Mair, ${ }^{2}$ Robert Hitchcock, ${ }^{4}$ Nik Hirani, ${ }^{2}$ \\ Chris Haslett, ${ }^{2}$ Mark Bradley $\mathbb{D}^{1},{ }^{1}$ and Kevin Dhaliwal $\mathbb{D}^{2}$ \\ ${ }^{1}$ EaStCHEM, The University of Edinburgh School of Chemistry, Joseph Black Building, West Mains Road, Edinburgh, UK EH9 3FJ \\ ${ }^{2}$ Translational Healthcare Technologies Group, Centre for Inflammation Research, Queen's Medical Research Institute, \\ University of Edinburgh, 47 Little France Crescent, Edinburgh BioQuarter, Edinburgh, UK EH16 4TJ \\ ${ }^{3}$ The Roslin Institute and Royal (Dick) School of Veterinary Studies, University of Edinburgh, Easter Bush, Midlothian, UK EH25 9RG \\ ${ }^{4}$ Department of Biomedical Engineering, University of Utah, 36 S Wasatch Dr, Salt Lake City, UT 84112, USA \\ ${ }^{5}$ School of Biomedical Engineering \& Imaging Sciences, King's College London, London, UK SE1 7EH \\ ${ }^{6}$ Mauna Kea Technologies, 9, Rue d'Enghien, Paris, France 75010
}

Correspondence should be addressed to Mark Bradley; mark.bradley@ed.ac.uk and Kevin Dhaliwal; kev.dhaliwal@ed.ac.uk

Alicia Megia-Fernandez, Adam Marshall, Ahsan R. Akram, and Bethany Mills contributed equally to this work.

Received 10 August 2020; Accepted 10 March 2021; Published 7 April 2021

Copyright ( 2021 Alicia Megia-Fernandez et al. Exclusive Licensee Suzhou Institute of Biomedical Engineering and Technology, CAS. Distributed under a Creative Commons Attribution License (CC BY 4.0).

Objective and Impact Statement. There is a need to develop platforms delineating inflammatory biology of the distal human lung. We describe a platform technology approach to detect in situ enzyme activity and observe drug inhibition in the distal human lung using a combination of matrix metalloproteinase (MMP) optical reporters, fibered confocal fluorescence microscopy (FCFM), and a bespoke delivery device. Introduction. The development of new therapeutic agents is hindered by the lack of in vivo in situ experimental methodologies that can rapidly evaluate the biological activity or drug-target engagement in patients. Methods. We optimised a novel highly quenched optical molecular reporter of enzyme activity (FIB One) and developed a translational pathway for in-human assessment. Results. We demonstrate the specificity for matrix metalloproteases (MMPs) 2, 9, and 13 and probe dequenching within physiological levels of MMPs and feasibility of imaging within whole lung models in preclinical settings. Subsequently, in a first-in-human exploratory experimental medicine study of patients with fibroproliferative lung disease, we demonstrate, through FCFM, the MMP activity in the alveolar space measured through FIB One fluorescence increase (with pharmacological inhibition). Conclusion. This translational in situ approach enables a new methodology to demonstrate active drug target effects of the distal lung and consequently may inform therapeutic drug development pathways.

\section{Introduction}

Respiratory diseases are one of the leading causes of morbidity and mortality worldwide [1]. Despite the significant burden of disease, there is a paucity of new pharmacological moieties reaching clinical phases of development and even fewer reaching clinical approval with spiraling costs of drug development [2]. Furthermore, the recent COVID-19 global pandemic has highlighted the need for rapid approaches to assess drugs that cause respiratory morbidity and mortality [3].

To date, the evaluation of early-phase clinical trials in human pulmonary disease has relied upon measurements, which include pulmonary function measurements, sixminute walk tests, and imaging such as computerized tomography (CT) [4]. These investigations can inform on functional and structural abnormalities but are delayed surrogates of active disease and offer little insight into 
dynamic molecular processes where therapeutics may be beneficial. Optical imaging modalities provide the potential for rapid readouts of disease activity at the site of pathogenesis, such as the distal lung [5]. Therefore, we developed a novel optical imaging approach using a combination of an existing miniaturized fibred confocal fluorescent microscopy (FCFM) system, a bronchoscope compatible delivery device, a new molecular probe, and a drug inhibitor to demonstrate real-time drug-target engagement in the distal lung of patients.

The matrix metalloproteinase (MMP) pathway was selected for detection and inhibition within a small patient population (eight patients with predicted aberrant lung fibrogenesis) to demonstrate the clinical tractability of our approach. MMPs are mediators of injury and repair, performing pivotal roles in the turnover of the extracellular matrix (ECM), with dysregulation leading to an aberrant response in inflammation and tissue repair, which ultimately leads to an impairment of organ function [6-9]. MMP dysregulation has been associated with many acute and chronic inflammatory respiratory diseases such as acute respiratory distress syndrome (ARDS) [10], idiopathic pulmonary fibrosis (IPF) [11], chronic obstructive pulmonary disease (COPD) [12], and lung cancer [13], with upregulation of the gelatinases (MMP-2 and MMP-9) often found in IPF $[9,14]$ and associated with a more aggressive disease phenotype [15]. The collagenase MMP-13 has also been shown to be abundant in the IPF tissue, [16] and as such, there has been considerable interest in the assessment of pulmonary MMP activity as a biomarker of fibrotic lung disease $[17,18]$ and in the therapeutic targeting of MMP activity in acute and chronic lung disease [19, 20] and the malignant matrix $[21,22]$.

The aim of this exploratory translational study was to develop and assess a novel optical reporter that was able to image MMP activity (measured though fluorescence increase of FIB One) within the alveolar space and demonstrate drugtarget engagement using a microdosing approach of a codelivered MMP inhibitor (moieties delivered being less than $100 \mu \mathrm{g}$ of imaging agent or drug). This methodology and the demonstration of real-time drug-target engagement in situ allowed the bedside assessment of pharmacological action in human disease using optical imaging.

\section{Results}

2.1. A Tribranched Scaffold Is Efficiently Cleaved by MMPs with Amplification of Fluorescence Signal within Minutes. We recently described a MMP peptide substrate $[23,24]$ that was selectively and specifically cleaved by secreted MMPs 2, 9, and 13 resulting in fluorescent signal amplification and designed to be resistant to plasmin and other bystander enzymes. However, although highly selective, this linear optimised probe demonstrated low signal-to-noise following cleavage.

To maximise the fluorescence amplification, we modified the linear probe by using a multiple Förster resonance energy transfer (FRET) system with insertion of the substrate peptide sequence into a tribranched scaffold. This tribranched structure takes advantage of both the self-quenching effect observed in multifluorophore constructs [25-27] as well as the quenching effect of three quenchers [28]. The molecular probe generated (called FIB One (Figure 1)) consisted of a tribranched peptide (Pro-Phe-Gly-Nle-Lys- $\beta \mathrm{Ala})_{3}$, containing three fluorescein units and three methyl red groups (Figure 1). FIB One was prepared using Fmoc-based solidphase peptide synthesis (Scheme S1 and ESI), and after purification, was fully characterized by reverse-phase highpressure liquid chromatography (RP-HPLC) and matrixassisted laser desorption ionising time-of-flight mass spectrometry (MALDI-ToF MS) (Figure 1(b) and ESI).

Selective hydrolysis of the peptidic sequence by the active enzyme results in an increase in fluorescence due to the release of the fragments containing fluorescein (Figure 1(c)), with excitation and emission maxima at 490/530 nm, respectively (Figure 1(d)). FIB One demonstrated rapid and specific fluorescent amplification when exposed to recombinant enzymes (MMPs 2, 9, and 13), which was blocked by the pan-MMP inhibitor marimastat (Figure 1(e) and Figure S1), with MALDI-ToF MS showing confirmation of selective cleavage (Figure S1). In addition, comparison of FIB One with its analogous monomeric linear structure confirmed improved signal amplification obtained with the multimeric approach (Figure S2).

\subsection{FIB One Is Cleaved by MMPs in Ex Vivo Primary Human} Models and in an Ex Vivo Lung Model. To determine the clinical tractability of this approach and for the compound to be cleaved at physiologically relevant/disease levels of MMPs, we evaluated the propensity for FIB One to be specifically cleaved by MMPs 2, 9, and 13 in primary human lung tissue and also by stimulated human neutrophils. FIB One was cleaved in both these scenarios with cleavage inhibition observed following coincubation with marimastat (Figures 2(a) and 2(b)). MALDI-ToF MS confirmed that the cleavage in these models was MMP specific (Figure S3).

The translational utility required evidence that the MMP activity could be rapidly detected in the distal lung in real time (within seconds to minutes) using an endoscopic approach. Therefore, we utilised an ex vivo ovine lung model (Figure 2(d)). Here, to demonstrate MMP upregulation, segments instilled with supernatant from spontaneously occurring ovine pulmonary adenocarcinoma (OPA) enabled evaluation of FIB One (Figure 2(c)). FCFM-based imaging of segments with high MMP activity (Figure S3) confirmed a diffuse pattern of increased fluorescence across the field of view, which was a characteristic of cleaved FIB One (Figure 2(d)). In contrast, vehicle control segments and marimastat (pan-MMP inhibitor)-dosed segments did not show an increase in FIB One signal (Figure 2(d)). Together, these data supported that pulmonary delivery of FIB One could detect MMP activity in situ when coupled with FCFM within a size relevant model within physiological concentrations.

2.3. GMP-Manufactured FIB One Demonstrated Stability, Lack of Toxicity, and Favourable Alveolar Imaging in Fibrotic Lung Disease in Human Patients. The translational pathway required toxicological and functional evaluation of FIB One. FIB One was synthesised in accordance with active pharmaceutical ingredient (API) development to 


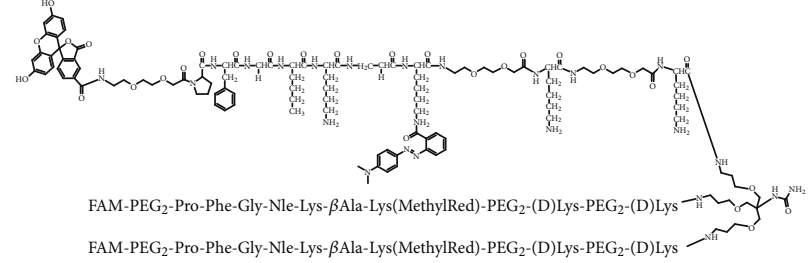

(a)

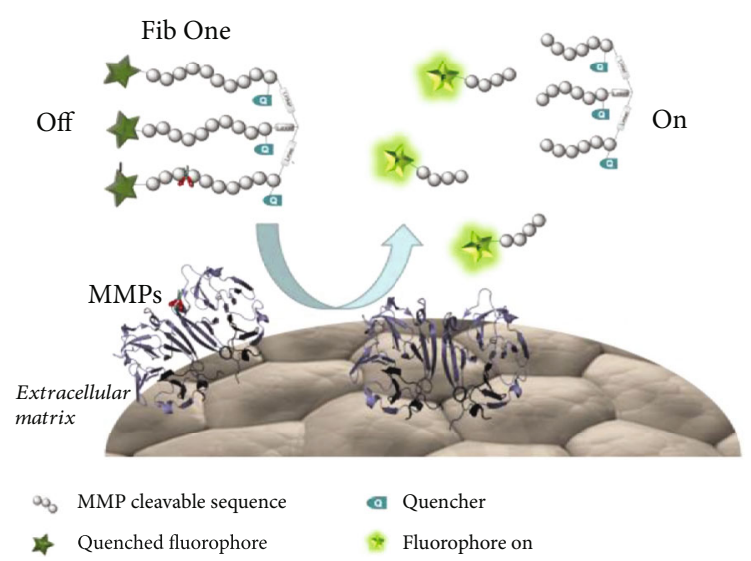

(c)

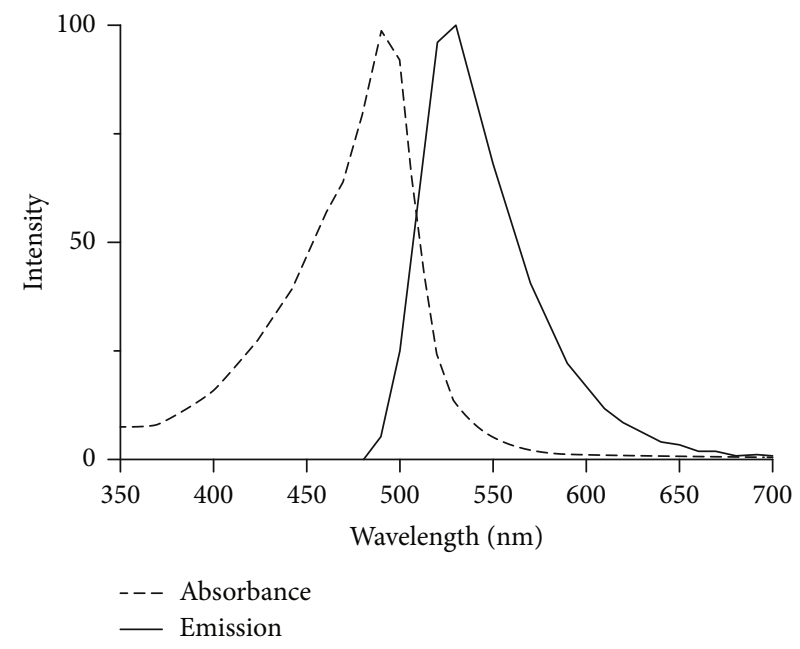

(d)

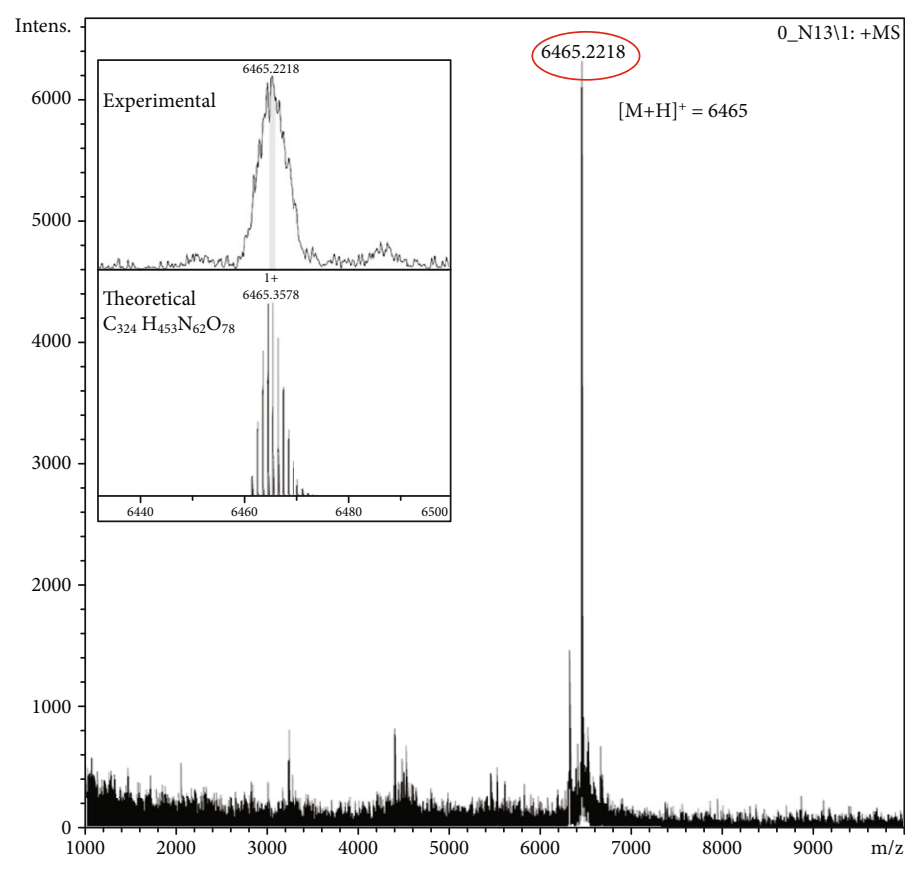

(b)

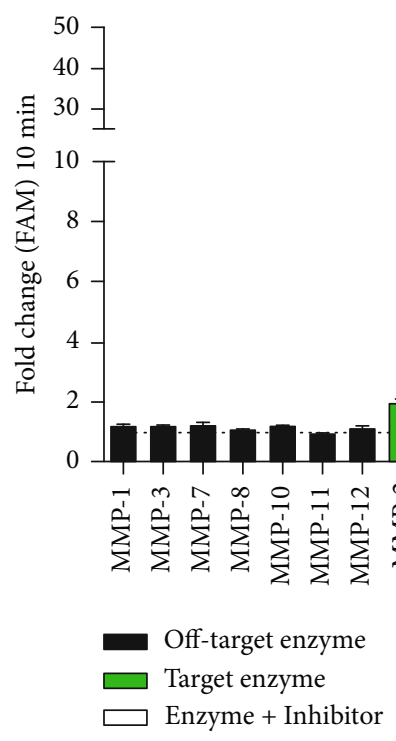

FIGURE 1: Structure, characterisation, mode of action, and in vitro validation of FIB One. (a) Chemical structure of FIB One. (b) FIB One characterisation by MALDI-ToF MS analysis: m/z $\left.6465[\mathrm{M}+\mathrm{H}]^{+}\right)$. (c) The FIB One structure showing how fluorescence switches from 'off' to 'on' following cleavage by the target MMP's. (d) Absorbance and emission spectra of FIB One. (e) Fold-change in fluorescent signal of FIB One $(1 \mu \mathrm{M})$ following $10 \mathrm{~min}$ incubation with target (green) and off-target (black) enzymes, compared to enzyme-free control. Activation of FIB One by target enzymes was prevented by the addition of the MMP inhibitor marimastat (20 $\mu \mathrm{M}$, white). Data shows mean and s.e.m. $n=3$ performed in duplicate, MMPs $30 \mathrm{nM}$, thrombin $5 \mathrm{U} / \mathrm{mL}$, plasmin $30 \mathrm{nM}$, factor Xa $500 \mathrm{nM}$, and excitation/emission $485 / 528 \mathrm{~nm}$.

good manufacturing practice (GMP) principles as stated in Eudralex [29]. It was prepared on a $100 \mathrm{mg}$ scale and demonstrated no degradation as an aqueous formulation over 12 months (Figure S4 and Table S8). FIB One demonstrated no overt biological toxicity, as evidenced by absence of erythrocyte hemolysis, and no preclinical in vivo toxicity, supported by a rodent intratracheal instillation model of high FIB One concentrations $(500 \mu \mathrm{g} / \mathrm{mL}$, compared to $20 \mu \mathrm{g} / \mathrm{mL}$ for intended human pulmonary dosing), which resulted in no pulmonary inflammatory cell 


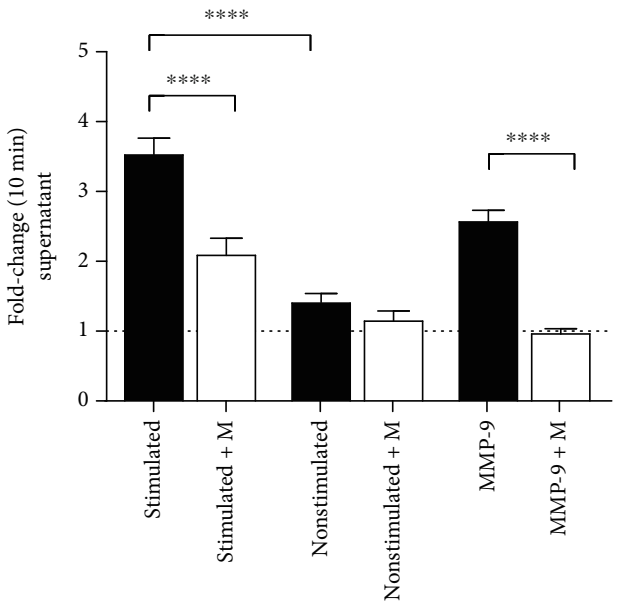

(a)

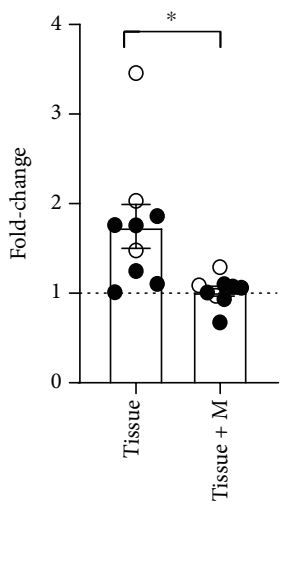

(b)

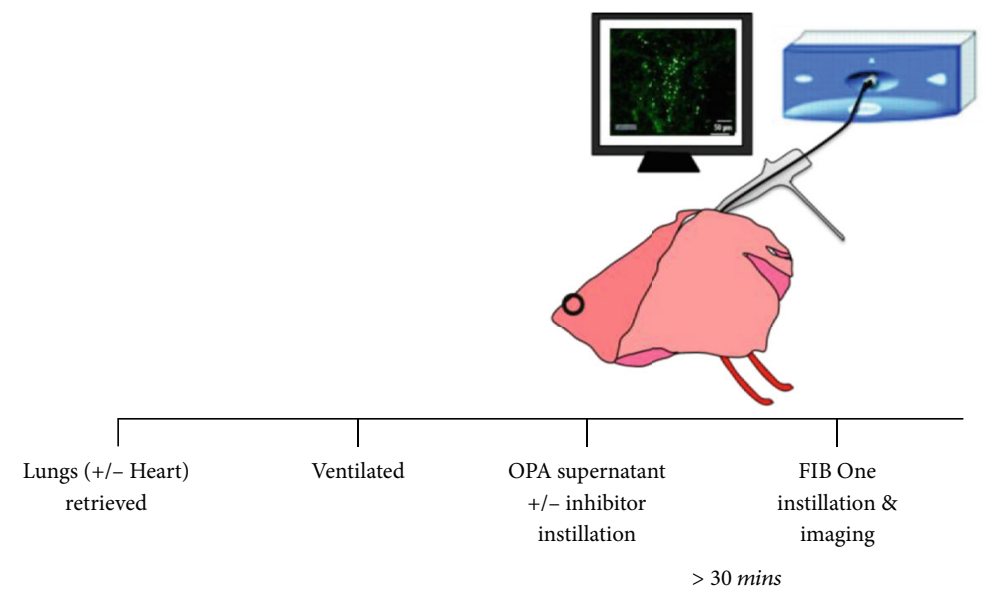

(c)
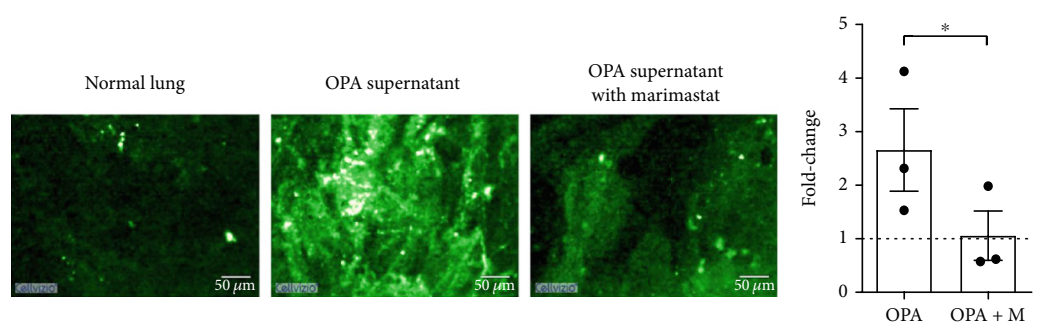

(d)

FIgURE 2: Validation of FIB One in physiologically relevant biological models. (a) Fold-change in FIB One (1 $\mu \mathrm{M})$ signal following 10 min incubation with supernatant from stimulated or nonstimulated neutrophils in vitro with and without a pan-MMP inhibitor, marimastat (M). Recombinant human MMP-9 (30 nM) shown as reference. Bars show mean (+/-SEM), $n \geq 4$ for each condition, analysis by one-way ANOVA ${ }^{* * * *} P<0.0001$. MALDI-ToF MS demonstrating site-specific probe cleavage in ESI Figure S3a. (b) Quantification from ex vivo fibre-based imaging of aged human lung tissue (open circles of pulmonary fibrosis patients and black circles of aged nonfibrotic lung tissue) with an FCFM imaging system ( $488 \mathrm{~nm}$ excitation). The fluorescence from samples with FIB One (1 $\mu \mathrm{M})$ or FIB One plus marimastat $(100 \mu \mathrm{M})$ was imaged $(10 \mathrm{~min})$ and quantified. Graph shows fold-change compared to baseline autofluorescence of the same sample. Data show mean (+/-SEM), $n=9,{ }^{*} P=0.0215$, student's paired $t$-test. (c) Experimental set-up of size-relevant ex vivo ovine pulmonary adenocarcinoma (OPA) sheep lung model. (d) Representative images of ex vivo ovine lung and OPA segments following administration of FIB One with and without inhibitor. Scale bar represents $50 \mu \mathrm{m}$. Graph demonstrates quantification of all experiments relative to intrinsic autofluorescence. $n=3$, bars show mean (+/-SEM), analysis by paired $t$-test for each normalised pre-post-FIB One segment, ${ }^{*} P=0.0476$. 

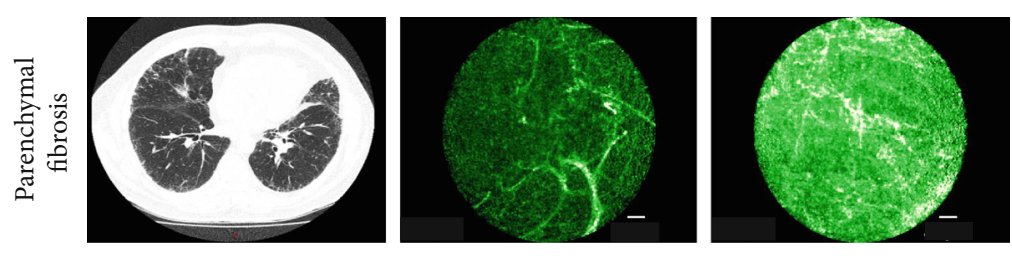

(a)

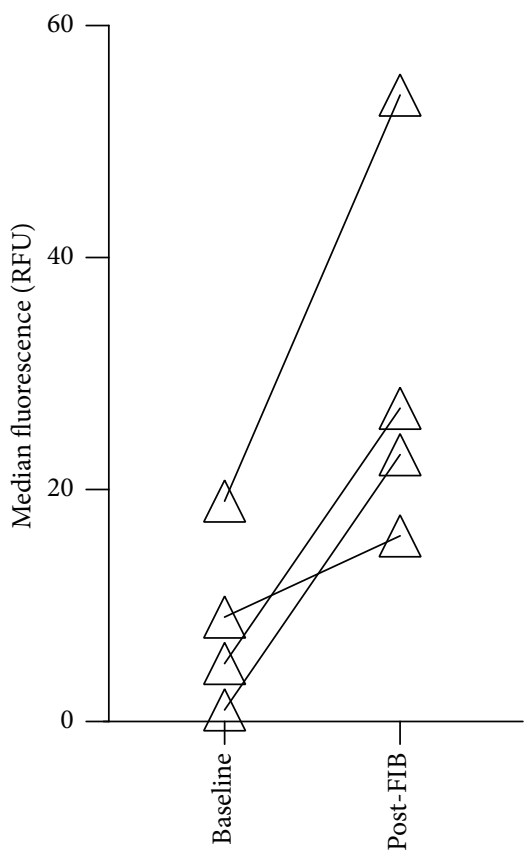

(b)

FIGURE 3: Imaging of parenchymal fibrosis following delivery of FIB One endobronchially. (a) Representative CT images (axial image from a patient with rheumatoid arthritis associated interstitial lung disease) with baseline and post-FIB One images acquired by confocal endomicroscopy imaging. (b) Quantification of in vivo human lung fluorescence measured using confocal endomicroscopy before and after bronchial delivery of the FIB One $(n=4$ for each group, $P>0.05)$. Statistical analysis using a Mann-Whitney test. Scale bar represents $50 \mu \mathrm{m}$.

recruitment, pulmonary toxicity, or systemic toxicity at either early or late time points (Table S7).

Using a conventional catheter in the working channel of a bronchoscope, we delivered FIB One endobronchially as a bolus to the lungs of eight patients with fibroproliferative lung disease in which we expected high MMP level (endobronchially visible tumours and diffuse pulmonary fibrosis). Alveolar FCFM imaging following endobronchial delivery of the probe resulted in increased fluorescent signal in all patients with parenchymal fibrosis (Figure 3). For cancer imaging, ex vivo data suggested that MMP activity utilising FIB One could be imaged but in vivo imaging of bronchial tumours was complicated by FIB One washing off the bronchial mucosa rapidly (Figure S5). There were no significant adverse events in the eight patients (Supplementary tables S1-S6).

\subsection{A Triple Lumen Bronchoscopy Catheter (TLBC) Enabled} Direct Imaging of MMP Activity in the Alveolar Space in Patients which Was Inhibited by Codelivery of a MMP Inhibitor. Although we detected MMP activity in patients with active fibrotic lung disease, our approach was hampered by requiring the delivery of a proximal bolus of FIB One down a conventional catheter in a step immediately preceding insertion of the imaging fibre. To enable concurrent FIB One delivery and imaging at the exact same location, we developed and deployed a triple lumen bronchoscopy catheter (TLBC) [30] which allows delivery of compounds and simultaneous FCFM imaging in the field of view.

To assess the distribution of agents delivered to the lung parenchyma, we undertook preclinical testing of the TLBC in an ex vivo ventilated human lung model. $500 \mu \mathrm{L}$ aliquots of iodinated contrast agent were delivered into the alveolar space and distribution assessed by CT scan, demonstrating a dispersal volume of $0.5 \mathrm{~cm}^{3}$ (Figure $4(\mathrm{a})$ ). The TLBC offered the capability to codeliver microdoses of a drug inhibitor. Thus, we reformulated an orally bioavailable small molecule inhibitor of MMPs (AZD1236) which had been previously evaluated in Phase-II trials into a GMP aqueous drug product and evaluated its toxicological and biological activity (Figure S6 Table S9). 

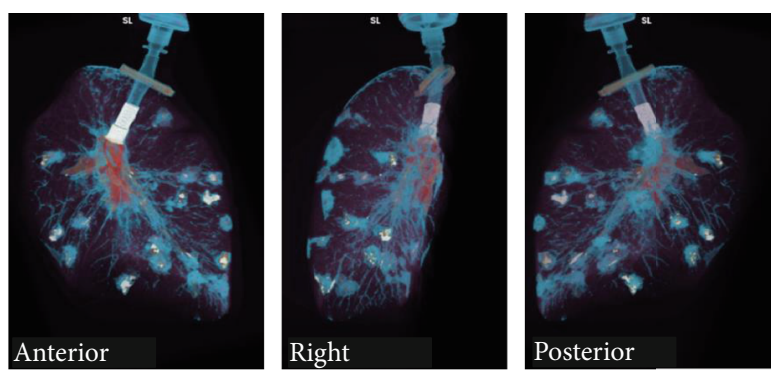

(a)
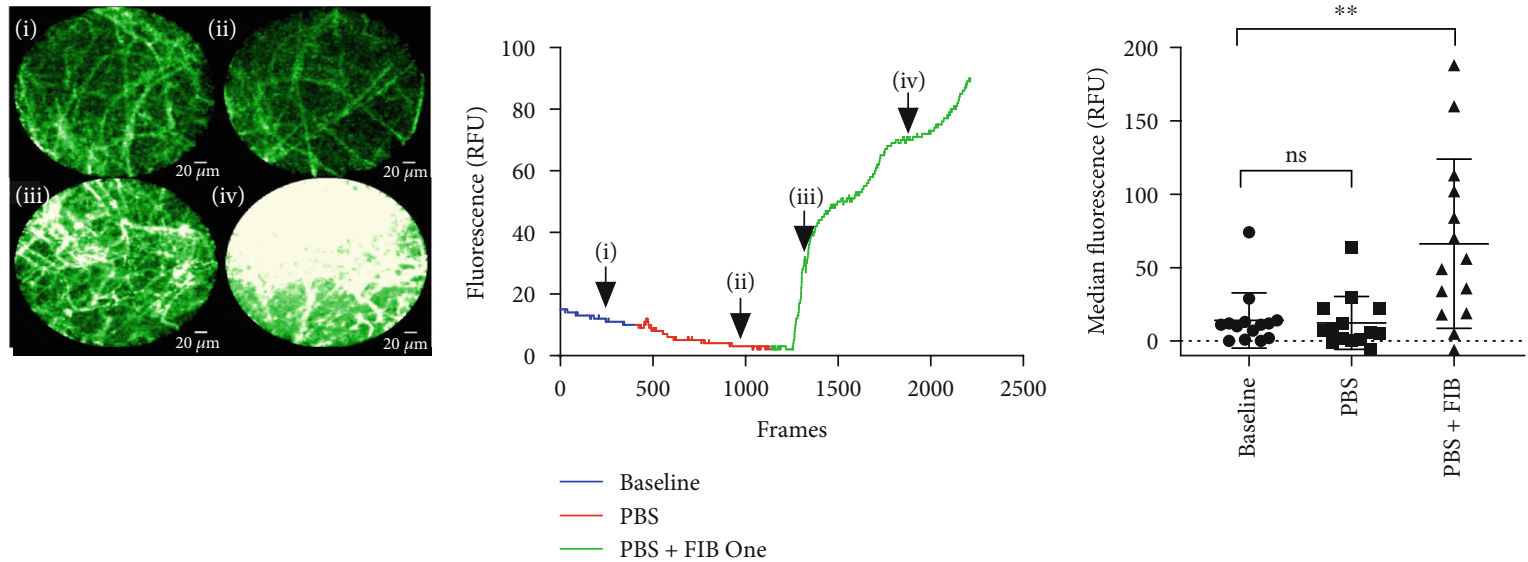

(b)

(c)

(d)
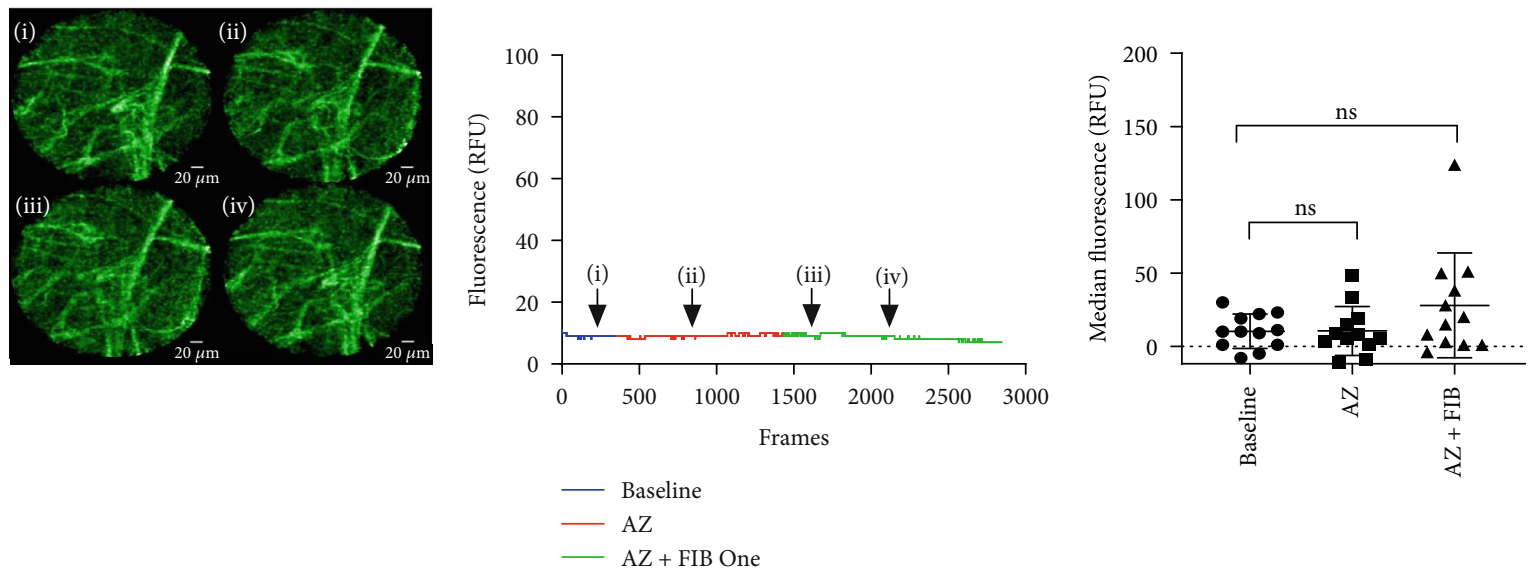

(e)

(f)

(g)

FIGURE 4: In vivo evidence of MMP activity with pharmacological inhibition in patients with pulmonary fibrosis. (a) CT images of an inflated ex vivo human right lung with $500 \mu \mathrm{L}$ aliquots of iodinated contrast delivered transbronchially. (b) Representative in vivo images from a patient with IPF: (i) alveolar space before administering any agents, (ii) after administering PBS, and (iii, iv) after the administration of FIB One. Dynamic range is identical for all images. Scale bar represents $20 \mu \mathrm{m}$. (c) Mean fluorescence of field of view over time during administration of PBS and FIB One. Corresponding time points to the images in (b) are indicated with arrows. (d) Median fluorescence of each video from 14 pulmonary segments $\left({ }^{\mathrm{ns}} P=0.36,{ }^{* *} P=0.002\right)$. Error bars represent mean $+/-$ s.d and statistical values from Wilcoxon matched pairs. (e) Representative images from a different pulmonary segment in the same patient as in (b): (i) alveolar space before administering any agents, (ii) after administering MMP inhibitor (AZD1236), and (iii, iv) after the administration of FIB One. Dynamic range is identical for all images. (f) Mean fluorescence of field of view over time during administration of AZD1236 and FIB One. Corresponding time points to the images in (e) are indicated with arrows. (g) Median fluorescence of each video from 12 pulmonary segments dosed with AZD1236 $(P=0.51)$ or AZD1236 plus FIB One $(P=0.05)$. Error bars represent mean $+/-$ s.d and statistical values from Wilcoxon matched pairs. RFU: relative fluorescence unit; PBS: phosphate-buffered saline; AZ: AZD1236 MMP inhibitor. 
Using this approach, we obtained real-time imaging sequences in nine patients with inflammatory fibroproliferative disease before and after FIB One delivery, with and without localised predelivery of AZD1236 (Figure 4, Table S5 and Figure S6), demonstrating in situ in vivo drug-target engagement within the alveolar space.

\section{Discussion}

We report the first in-human pulmonary optical molecular imaging of enzyme activity, through fluorescence increase of an MMP reporter, and dynamic elucidation of drugtarget engagement in the distal alveolar regions of the human lung.

The FIB One probe was specific to MMPs 2, 9, and 13 which degrade gelatin, type IV collagen, and elastin within the ECM, which are known to be abundant in the context of acute and chronic inflammation [31] where they are secreted by infiltrating inflammatory cells as well as bronchial and alveolar epithelial cells and fibroblasts [32]. To ensure future validity of our work, there would need to be inclusion of a noninflammatory control group to determine the detection of upregulated vs. homeostatic MMP activity. However, if confirmed, MMPs represent an attractive target as they serve as a marker in several human inflammatory diseases including IPF, COPD, ARDS, and lung cancer [10-14]. With the advent of increasingly available multiplexing approaches for microscopic interrogation, our approach of using fluorescent reporters with FCFM and drug inhibition does represent a wider applicability and is readily extendable to multiple target pathways in several organ systems.

Current approaches to identifying enzyme activity in the lung has been challenging and has typically been performed ex vivo using zymography on patient tissue or lavage samples [33]. However, this technique has several limitations, including the requirement for an invasive biopsy and tissue retrieval and the utilisation of lavage fluid, which is prone to sampling inconsistencies. Furthermore, zymography assessment may not reflect free enzyme activity in the native environment, which in the case of MMPs, is dependent on inhibitory antagonists such as tissue inhibitors of MMPs (TIMPs).

Molecular imaging of enzyme activity and pharmacological perturbation in vivo has focused on either nuclear imaging of radiolabelled probes or optical imaging with fluorogenic probes. These studies have used animal models to image MMP activity in vivo in cancer [34-39], stroke [40], and joint disease [41-43]; however, they have all relied on systemic intravenous delivery of the probes and wholebody optical imaging, which is to date, technically not possible in humans with sufficient resolution or sensitivity. Thus, we adopted an alternate approach to measure enzyme activity in the distal lung human lung, made possible by using FCFM to allow us to access alveolar regions and deliver microdoses $(<100 \mu \mathrm{g})$ of FIB One directly to the region of interest without concerns of toxicity. It was of importance that the area of tissue imaged coincided with the delivery of the imaging reagent (FIB One). Whilst our initial endobronchial delivery yielded encouraging results, we could not elim- inate the possibility that FCFM fibre placement would miss a predosed alveolar region. Our preclinical data supports the use of this approach in lung cancer, although there were technical difficulties with imaging endobronchial tumours with rapid dissipation of probe. These may be mitigated in the future with further adaptation to the technical approach or modifications to the probe to allow cancer cell labelling [24].

MMP inhibition has been identified as a target for drug interventions in inflammatory diseases [44, 45]. MMP inhibitors used in trials as cancer therapies were disappointing due to lack of efficacy and significant adverse effects [46]. Studies have also been limited by a lack of quantifiable biomarkers to reflect on their in vivo and functional activity [20]. In this study, we reformulated and toxicologically qualified AZD1236, an orally available MMP-9/12 inhibitor which also demonstrated an ability to inhibit MMPs 2 and 13. It had previously been used in a phase $2 \mathrm{~A}$ safety and tolerability study in 74 emphysema patients randomised to placebo or AZD1236 $75 \mathrm{mg}$ twice daily for 6 weeks [19]. The study demonstrated no effect on the secondary lung physiology endpoints, and the investigators were unable to show that AZD1236 actually inhibited its intended targets in situ. Indeed, a placebo-controlled, AZD1236 biomarker study of 55 COPD patients again failed to demonstrate MMP-9 or MMP-12 inhibition in induced sputum [20], whereas we have demonstrated drug-target engagement of AZD1236 in humans.

The pathway of drug validation utilises cell-based assays and animal models of disease followed by phased studies in humans. At each stage of development, costs increase and there is a high attrition rate often leading to the failure or deescalation of drug candidates. Although there are many reasons for this, one major hurdle is demonstrating that enzymatic activity is present in the patients being studied. Therefore, there is a rationale for performing translational proof of concept (experimental medicine) studies directly and rapidly in humans, using small numbers of patients. Consequently, here, we have described a tractable strategy for rapidly assessing distal alveolar biology using a sensitive and specific optical probe and direct visualisation of MMP pathway activity/inhibition in situ in real time with FCFM. In the future, this could be assessed in a broader group of patients with inflammatory and noninflammatory lung disease correlated with the MMP activity and may present the ability to offer a mechanism-based method of stratifying patients for drug studies and to interrogate biological pathways in situ in vivo with molecular microscopy.

\section{Materials and Methods}

4.1. Ethics Statement. All experiments using human samples in vitro were performed following approval of the appropriate regional ethics committee (REC) and with informed consent of the patients. Blood sample collection and use was approved by Lothian REC No: 08/S1103/38 and $15 / \mathrm{HV} / 013$. Tissue samples were obtained following approval by NHS Lothian REC and facilitated by NHS Lothian SAHSC Bioresource REC No: 13/ES/0126 and NHS Lothian REC No: 09/S1101/52. The ex vivo human lungs 
were deemed unsuitable for transplant and authorised for research as part of the Enlighten study (London-Central Research Ethics Committee, REC Reference: 16/LO/1883). The in-human assessments were undertaken with informed consent of the participants and approved by the South East Scotland Regional Ethics Committee 02, REC Number: 15/SS/0235. The trial was registered with ClinicalTrials.gov (Identifier: NCT02604862).

4.2. In Vitro Enzymatic Validation of FIB One. To determine the enzymatic specificity of FIB One, the probe was incubated at a concentration of $1 \mu \mathrm{M}$ (unless otherwise stated) with the recombinant MMPs (Enzo Life Sciences) (active domains of MMP-1, MMP-2, MMP-3, MMP-7, MMP-8, MMP-9, MMP-10, MMP-11, MMP-12, and MMP-13, all at $30 \mathrm{nM}$ ), thrombin (Sigma Aldrich) $(5 \mathrm{U} / \mathrm{mL})$, factor Xa (Sigma Aldrich) (500 nM), plasmin (Sigma Aldrich) $(30 \mathrm{nM})$, and human neutrophil elastase $(30 \mathrm{nM})$. Where appropriate, enzymes were incubated with specific inhibitors for $30 \mathrm{~min}$ at $37^{\circ} \mathrm{C}$ prior to addition of FIB One. The pan-MMP inhibitor marimastat (Tocris Biosciences) was used at $20 \mu \mathrm{M}$, and the MMP inhibitor AZD1236 was used at 0-14 $\mu \mathrm{M}$. Enzyme free reactions served as a control. Enzymatic reactions were performed in MMP buffer $\left(10 \mathrm{mM} \mathrm{CaCl}_{2}, 6.1 \mathrm{~g}\right.$ Tris- $\mathrm{HCl}$, $8.6 \mathrm{~g} \mathrm{NaCl}$ per litre, $\mathrm{pH} 7.5$ ) in a final volume of $20 \mu \mathrm{L}$. Reactions were performed in duplicate in 384-well plates (Life Technologies) with optically clear plate seals (Thermo Scientific) and repeated thrice (independently). Fluorescence signal was measured for up to $60 \mathrm{~min}$, ex/em $485 / 528 \mathrm{~nm}$ using microplate reader (BioTek Synergy $\mathrm{H} 1$ multimode reader). Data were normalised to buffer alone and are presented as fold-change in signal (relative fluorescent units, RFU) compared to enzyme-free controls. Data were plotted using Prism 7 (GraphPad Software Inc., La Jolla, CA, USA).

4.3. Neutrophil Extraction and Evaluation of FIB One. Neutrophils were isolated from the blood of healthy human volunteers as previously described [47]. The number of retrieved neutrophils was determined with NucleoCounter NC-1000 (Chemo Metec). Neutrophils were resuspended at a concentration of $20 \times 10^{6} \mathrm{~mL}^{-1}$ in $0.9 \% \mathrm{NaCl}$ with $0.9 \mathrm{mM}$ $\mathrm{CaCl}_{2}$. Cells were incubated for $30 \mathrm{~min}$ at $37^{\circ} \mathrm{C}$. Cells to be stimulated were subsequently activated with $5 \mu \mathrm{M}$ calcium ionophore A23187 (Tocris Bioscience) for $30 \mathrm{~min}$ at $37^{\circ} \mathrm{C}$. Neutrophils were harvested by centrifugation at $400 \mathrm{x} g$ for $5 \mathrm{~min}$. Supernatants were removed and stored. Plate reader assays were performed as described above, with the MMP buffer replaced with neutrophil supernatant. All experiments were carried out in duplicate using three independent donors (one donor per independent repeat). Data were normalised by background subtraction of intrinsic fluorescence.

4.4. Ex Vivo Human Lung Tissue Processing and Evaluation of FIB One. Human lung tissue samples (lung tumour and noncancerous adjacent tissue) were obtained following surgical resection and stored at $-80^{\circ} \mathrm{C}$ until use. Processing for multiwell plate reader assay or zymography analysis was conducted as follows: $1 \mathrm{~mm} \times 4 \mathrm{~mm}$ sections were homogenised in $400 \mu \mathrm{L}$ MMP buffer in Precellys $2.8 \mathrm{~mm}$ ceramic bead tubes (VWR) using a Pecellys 24 homogeniser. Homogenised tissue was adjusted to $4 \mathrm{mg} \mathrm{mL}^{-1}$ protein (determined by a Pierce BCA Total Protein Assay Kit (Thermo Fisher Scientific), following the manufacturer's instructions). Multiwell plate reader assays were performed as previously described, replacing the MMP buffer and MMP enzymes for the homogenised and protein-adjusted lung tissue.

For zymography, the samples were mixed $1: 1$ with $2 x$ SDS sample buffer and $20 \mu \mathrm{L}$ was loaded onto precast Novex ${ }^{\circledR}$ 10\% Zymogram (Gelatin) Protein Gels (Thermo Fisher Scientific). The gels were placed in an electrophoresis chamber with pre-chilled Novex diluted running buffer and electrophoresed at $150 \mathrm{~V}$ for approximately $90 \mathrm{~min}$ at $4^{\circ} \mathrm{C}$. Gels were removed and incubated with Novex renaturing buffer for $90 \mathrm{~min}$ at $4^{\circ} \mathrm{C}$. They were washed in distilled water and incubated with Novex developing buffer for $30 \mathrm{~min}$ at room temperature prior to overnight incubation at $37^{\circ} \mathrm{C}$. Control gels had marimastat $(50 \mu \mathrm{M})$ added to the developing buffer. Gels were then rinsed with distilled water prior to staining with a colloidal blue staining kit and imaging using a transilluminator (UVItec BXT-20 M, UVItec Ltd, Cambridge, UK).

For ex vivo imaging, human lung tissue was sliced into $1 \mathrm{~mm} \times 4 \mathrm{~mm}$ sections and placed in the wells of a 96-well plate with $100 \mu \mathrm{L}$ of MMP buffer and $30 \mathrm{nM}$ MMP-13. Where appropriate, AZD1236 $(0-14 \mu \mathrm{M}$ as indicated in the text) was added to the tissue and incubated at $37^{\circ} \mathrm{C}$ for 30 min prior to the addition FIB One $(5 \mu \mathrm{M})$. The tissue was imaged over $10 \mathrm{~min}, 12$ frames $\sec ^{-1}$ using a preclinical confocal laser scanning endomicroscopy device (Cellvizio ${ }^{\circledR}$, Mauna Kea Technologies, excitation $488 \mathrm{~nm}$ /detection bandwidth 505 to $700 \mathrm{~nm}$ ) with a compatible imaging fibre (Alveoflex ${ }^{\mathrm{TM}}$, Mauna Kea Technologies). At each time point $60 \mathrm{~s}$, images of captured with same laser power. The fluorescence for each sample was calculated by determining the average fluorescence per frame for each time point. All images were brightness and contrast enhanced with the same parameters. Five independent patient specimens were collected and analysed. RFUs collected from off-target frames (showing motion artefact or absence of any fluorescence) were omitted during analysis.

4.5. Ex Vivo Lung Experimental Procedure. Ovine pulmonary adenocarcinoma (OPA) tissue was homogenised using a drill homogeniser, followed by lysis in cell lysis buffer. The supernatant was collected following centrifugation at $12,000 \mathrm{rpm}$ for $20 \mathrm{~min}$. The samples were adjusted to $1 \mathrm{mg} \mathrm{mL}^{-1}$ total protein (determined by a Pierce BCA Total Protein Assay Kit (Thermo Fisher Scientific)). Zymography (as described above) was performed to confirm MMP activity across multiple OPA samples. Recombinant MMP-9 (2 nM) and recombinant pro-MMP-9 $(0.5 \mathrm{nM})$ were included in the assay as positive controls.

OPA supernatant $(1 \mathrm{~mL})$ and marimastat $(100 \mu \mathrm{M})$ or $1 \mathrm{~mL} 0.9 \% \mathrm{NaCl}$ (control) was instilled into anatomically distinct segments of ex vivo ventilated non-OPA ovine lungs. Following $>30 \mathrm{~min}$, the segments were bronchoscopically identified and microdosed with $5 \mu \mathrm{M}$ FIB One in $1 \mathrm{~mL}$. FCFM imaging was undertaken pre- and postinstillation 
(Cellvizio ${ }^{\circledR}$, Mauna Kea Technologies). This was performed by passing a FCFM fibre into the disparate bronchopulmonary segments (up to 5 passes per bronchopulmonary segment to capture a regional representation of fluorescence), recording images at 12 frames per second and imaging for up to 5 minutes. Videos were analysed as described above. Each experimental set was normalised to the control segment (segment with $1 \mathrm{~mL} 0.9 \% \mathrm{NaCl}$ instilled) which represents intrinsic autofluorescence.

4.6. Human Ex Vivo Experimental Procedure. The development of the TLBC has been described previously [30]. This device was compatible with a slimmer (diameter $1 \mathrm{~mm}$ ) imaging fibre approved for clinical investigation (Miniaturized AlveoFlex ${ }^{\mathrm{TM}}$ Confocal Miniprobe, Cellvizio ${ }^{\circledR}$, Mauna Kea Technologies) and had two hollow lumens to allow FIB One and/or AZD1236 drug delivery directly to tissue within the field of view during image acquisition. The TLBC device was validated preclinically within ventilated ex vivo human lungs to assess both fluid delivery capability and to determine which segments of the lung the device was able to reach. Iodinated contrast (Omnipaque 300, GE healthcare) was diluted with PBS in a ratio of $1: 5$ and delivered transbronchially in $500 \mu \mathrm{L}$ doses through the device lumens to the distal lung, repeated for multiple pulmonary segments. Following delivery, the lung was inflated, the trachea clamped, and computerized tomography (Biograph mCT, Siemens) images were taken to visualise the extent of alveolar contrast spread. Processing was performed with Horos DICOM viewer (HOROS).

4.7. Clinical Study Patient Selection. All participants were scheduled for routine, clinical diagnostic flexible bronchoscopy in our institution with a known or suspected diagnosis of fibrosis or lung cancer. All eight patients had computerized tomography performed prior to bronchoscopy and regions of active disease identified for targeted investigation.

4.8. Bronchoscopy and FCFM Procedure. Bronchoscopy was undertaken in an outpatient suite using local anaesthetic and conscious sedation or in theatre under general anaesthetic. FCFM was performed using a clinically approved confocal laser scanning endomicroscopy device (Cellvizio ${ }^{\circledR}$, Mauna Kea Technologies) with a compatible imaging fibre Alveoflex $^{\mathrm{TM}}$ or Miniaturized Alveoflex ${ }^{\mathrm{TM}}$, Mauna Kea Technologies).

In initial exploratory testing, 8 patients with either endobronchial tumour or parenchymal fibrosis underwent FIB One microdosing $(<100 \mu \mathrm{g},<5 \mathrm{~mL})$. FIB One was administered using a delivery catheter (ERBE APC catheter or TLBC) passed out of the working channel of the wedged bronchoscope into the bronchus allowing distal dissipation of the compound for alveolar imaging or applied directly onto visible tumour. FCFM imaging was performed pre- and postinstillation of FIB One, with the fibre tip positioned at the bronchial mucosa or passed into the alveolar region following transbronchial puncture, as appropriate for the clinical indication. Images were recorded for at least $30 \mathrm{~s}$ at 12 frames/second.
4.9. In Vivo Assessment of FIB One and AZD1236 with TLBC. In subsequent testing the Miniaturized Alveoflex ${ }^{\mathrm{TM}}-\mathrm{TLBC}$ combination replaced the Alveoflex ${ }^{\mathrm{TM}}$ fibre. The entire apparatus was passed transbronchially to allow direct alveolar imaging before localised delivery of agents. Once transbronchial pass was accomplished, baseline lung imaging was recorded for at least $30 \mathrm{sec}$. A $500 \mu \mathrm{L}$ microdose of either AZD1236 $(14 \mu \mathrm{g} / \mathrm{mL})$ or sterile PBS (control) was administered under direct observation, and further imaging was obtained for $>60 \mathrm{sec}$. $500 \mu \mathrm{L}$ of FIB One $(20 \mu \mathrm{g} / \mathrm{mL}$ ) (delivered only after at least 60 s to allow MMP inhibition) was then dosed to the same area with a further $90-120 \mathrm{sec}$ of imaging captured. If considerable movement due to breathing or coughing was encountered, imaging was halted and a new segment identified. Up to 6 segments of interest were investigated per patient determined by patient tolerance.

4.10. Image Analysis. All video sequences were assessed by two clinicians with clinical experience of pulmonary endomicroscopy. Frames with excessive movement or lack of recognizable alveolar structure were removed from analysis. Videos were assessed to ensure stability throughout the imaging procedure. To ensure the region imaged was the same as region dosed, imaging from a segment was excluded if there was obvious alveolar movement witnessed during image capture. All video files were analysed using the Cellvizio ${ }^{\circledR}$ Viewer image processing software from Mauna Kea Technologies, Paris. A region of interest was drawn around the fibre field of view and processed to determine the mean RFU for each frame. The videos were categorized as either baseline, postPBS, post-AZD1236, post-FIB One, and PBS or post-FIB One and AZD1236 for each patient. The median RFU was calculated for each category and used as a comparative value between groups.

4.11. Statistical Analysis. Statistical analyses were performed using Prism 7 (GraphPad Software Inc., La Jolla, CA, USA). Where appropriate, analyses were performed using the student's $t$-test or one-way ANOVA. Unless otherwise stated error bars show standard error of the mean (s.e.m).

\section{Conflicts of Interest}

$\mathrm{KD}, \mathrm{CH}$, and $\mathrm{MB}$ are shareholders of Edinburgh Molecular Imaging. MB, SVC, and AMF are inventors on a patent (WO 2016/151299 A1) held by the University Court of the University of Edinburgh that covers the probe and method of use. FL and VD are employees of Mauna Kea Technologies. TV owns stock from Mauna Kea Technologies. The other authors declare that they have no competing interests.

\section{Authors' Contributions}

AMF, SVC, and MB performed the chemical design, synthesis, and characterisation. AA, BM, AMi, CM, BCM, and KD designed and performed the in vitro biological assays. AMa, $\mathrm{AA}$, and $\mathrm{KD}$ designed, set-up, and performed the ex vivo lung experiments. ES, AB, JM, AA, AMa, NH, and KD set-up, recruited, and undertook the clinical studies and undertook data analysis. RH, NK, TV, AB, JM, FL, VD, AA, and KD 
developed the TLBC. $\mathrm{CH}, \mathrm{MB}$, and $\mathrm{KD}$ supervised the project. AMF, AA, AMa, BM, and $\mathrm{KD}$ wrote the manuscript. All authors approved the manuscript. Alicia MegiaFernandez, Adam Marshall, Ahsan R. Akram, and Bethany Mills contributed equally to this work.

\section{Acknowledgments}

The authors would like to thank the NHS Lothian SAHSC Bioresource for facilitating the studies using ex vivo human lung tissue, Dr Chris Cousins for the samples of OPA lung, AstraZeneca UK for providing AZD1236 Drug Product as an in-kind contribution through their Open Innovation Program, Warren's Wish (Registered Scottish Charity: SC 045290), and the human lung donor and their families. This study was supported by the Medical Research Council (under the Developmental Pathway Funding Scheme grant number MR/J014702), the Engineering and Physical Sciences Research Council (EP/K03197X/1, EP/R005257/1, and NS/A000049/1), and the Wellcome Trust (203148/Z/16/Z). AA is supported by a Cancer Research UK Clinician Scientist Fellowship (A24867). TV is supported by a Medtronic/Royal Academy of Engineering Research Chair (RCSRF1819\7\34). The research leading to these results has received funding from the European Union Seventh Framework Programme FP7 2012 under grant agreement No. 326465 (AMF). Mauna Kea Technologies provided support for the clinical development of the TLBC.

\section{Supplementary Materials}

Supplementary Information containing supplementary Figures S1-6: chemistry materials and methods, patient data, toxicology studies, and stability studies. (Supplementary Materials)

\section{References}

[1] H. Wang, M. Naghavi, C. Allen et al., "Global, regional, and national life expectancy, all-cause mortality, and causespecific mortality for 249 causes of death, 1980-2015: a systematic analysis for the Global Burden of Disease Study 2015," The Lancet, vol. 388, no. 10053, pp. 1459-1544, 2016.

[2] G. Khurana, A. Rohilla, and A. Deep, "Drug development process and novel drugs approved by FDA for 2017-18," Applied Clinical Research, Clinical Trials and Regulatory Affairs, vol. 5, no. 2, pp. 80-98, 2018.

[3] B. N. Rome and J. Avorn, "Drug evaluation during the Covid19 pandemic," New England Journal of Medicine, vol. 382, no. 24, pp. 2282-2284, 2020.

[4] P. Spagnolo, E. Cocconcelli, and V. Cottin, "Clinical trials in IPF: what are the best endpoints?," in Idiopathic Pulmonary Fibrosis: A Comprehensive Clinical Guide, K. C. Meyer and S. D. Nathan, Eds., pp. 433-453, Springer International Publishing, Cham, 2019.

[5] A. R. Akram, S. V. Chankeshwara, E. Scholefield et al., "In situ identification of Gram-negative bacteria in human lungs using a topical fluorescent peptide targeting lipid A," Science Translational Medicine, vol. 10, no. 464, article eaal0033, 2018.
[6] D. C. Rockey, P. D. Bell, and J. A. Hill, "Fibrosis - a common pathway to organ injury and failure," New England Journal of Medicine, vol. 372, no. 12, pp. 1138-1149, 2015.

[7] S. D. Shapiro, "Matrix metalloproteinase degradation of extracellular matrix: biological consequences," Current Opinion in Cell Biology, vol. 10, no. 5, pp. 602-608, 1998.

[8] M. G. Rohani and W. C. Parks, "Matrix remodeling by MMPs during wound repair," Matrix Biology, vol. 44-46, pp. 113-121, 2015.

[9] M. Corbel, C. Belleguic, E. Boichot, and V. Lagente, "Involvement of gelatinases (MMP-2 and MMP-9) in the development of airway inflammation and pulmonary fibrosis," Cell Biology and Toxicology, vol. 18, no. 1, pp. 51-61, 2002.

[10] A. Davey, D. F. McAuley, and C. M. O'Kane, "Matrix metalloproteinases in acute lung injury: mediators of injury and drivers of repair," European Respiratory Journal, vol. 38, no. 4, pp. 959-970, 2011.

[11] A. Pardo and M. Selman, "Role of matrix metaloproteases in idiopathic pulmonary fibrosis," Fibrogenesis \& Tissue Repair, vol. 5, Supplement 1, p. S9, 2012.

[12] L. Segura-Valdez, A. Pardo, M. Gaxiola, B. D. Uhal, C. Becerril, and M. Selman, "Upregulation of gelatinases A and B, collagenases 1 and 2, and increased parenchymal cell death in COPD," Chest, vol. 117, no. 3, pp. 684-694, 2000.

[13] S. A. Shah, F. G. Spinale, J. S. Ikonomidis, R. E. Stroud, E. I. Chang, and C. E. Reed, "Differential matrix metalloproteinase levels in adenocarcinoma and squamous cell carcinoma of the lung," The Journal of Thoracic and Cardiovascular Surgery, vol. 139, no. 4, pp. 984-990, 2010.

[14] A. Pardo, S. Cabrera, M. Maldonado, and M. Selman, "Role of matrix metalloproteinases in the pathogenesis of idiopathic pulmonary fibrosis," Respiratory Research, vol. 17, no. 1, p. 23, 2016.

[15] M. Selman and A. Pardo, "Revealing the pathogenic and agingrelated mechanisms of the enigmatic idiopathic pulmonary fibrosis. An integral model," American Journal of Respiratory and Critical Care Medicine, vol. 189, no. 10, pp. 1161-1172, 2014.

[16] T. Nkyimbeng, C. Ruppert, T. Shiomi et al., "Pivotal role of matrix metalloproteinase 13 in extracellular matrix turnover in idiopathic pulmonary fibrosis," PLoS One, vol. 8, no. 9, article e73279, 2013.

[17] R. G. Jenkins, J. K. Simpson, G. Saini et al., "Longitudinal change in collagen degradation biomarkers in idiopathic pulmonary fibrosis: an analysis from the prospective, multicentre PROFILE study," The Lancet Respiratory Medicine, vol. 3, no. 6, pp. 462-472, 2015.

[18] B. Ley, K. K. Brown, and H. R. Collard, "Molecular biomarkers in idiopathic pulmonary fibrosis," American Journal of Physiology-Lung Cellular and Molecular Physiology, vol. 307, no. 9, pp. L681-L691, 2014.

[19] H. Magnussen, H. Watz, A. Kirsten et al., "Safety and tolerability of an oral MMP-9 and -12 inhibitor, AZD1236, in patients with moderate-to-severe COPD: A randomised controlled 6week trial," Pulmonary Pharmacology \& Therapeutics, vol. 24, no. 5, pp. 563-570, 2011.

[20] R. Dahl, I. Titlestad, A. Lindqvist et al., "Effects of an oral MMP-9 and -12 inhibitor, AZD1236, on biomarkers in moderate/severe COPD: a randomised controlled trial," Pulmonary Pharmacology \& Therapeutics, vol. 25, no. 2, pp. 169-177, 2012. 
[21] K.-H. Shen, J. H. Hung, C. W. Chang, Y. T. Weng, M. J. Wu, and P. S. Chen, "Solasodine inhibits invasion of human lung cancer cell through downregulation of miR-21 and MMPs expression," Chemico-Biological Interactions, vol. 268, pp. 129-135, 2017.

[22] P. D. Brown, "Matrix metalloproteinase inhibitors in the treatment of cancer," Medical Oncology, vol. 14, no. 1, pp. 1-10, 1997.

[23] A. Megia-Fernandez, B. Mills, C. Michels et al., "Bimodal fluorogenic sensing of matrix proteolytic signatures in lung cancer," Organic \& Biomolecular Chemistry, vol. 16, no. 43, pp. 8056-8063, 2018.

[24] B. Mills, D. Norberg, K. Dhaliwal, A. R. Akram, M. Bradley, and A. Megia-Fernandez, "A matrix metalloproteinase activation probe for painting human tumours," Chemical Communications, vol. 56, no. 69, pp. 9962-9965, 2020.

[25] N. Avlonitis, M. Debunne, T. Aslam et al., "Highly specific, multi-branched fluorescent reporters for analysis of human neutrophil elastase," Organic \& Biomolecular Chemistry, vol. 11, no. 26, pp. 4414-4418, 2013.

[26] A. K. Galande, S. A. Hilderbrand, R. Weissleder, and C. H. Tung, "Enzyme-targeted fluorescent imaging probes on a multiple antigenic peptide core," Journal of Medicinal Chemistry, vol. 49, no. 15, pp. 4715-4720, 2006.

[27] J. M. Ellard, T. Zollitsch, W. J. Cummins, A. L. Hamilton, and M. Bradley, "Fluorescence enhancement through enzymatic cleavage of internally quenched dendritic peptides: a sensitive assay for the AspN endoproteinase," Angewandte Chemie (International Ed. in English), vol. 41, no. 17, pp. 3233-3236, 2002.

[28] T. H. Craven, N. Avlonitis, N. McDonald et al., "Super-silent FRET sensor enables live cell imaging and flow cytometric stratification of intracellular serine protease activity in neutrophils," Scientific Reports, vol. 8, no. 1, article 13490, 2018.

[29] EU, "EU guidelines for good manufacturing practice for medicinal products for human and veterinary use," vol. 4, Heath and Consumers Directorate-General, European Comission, 2014, Part II.

[30] N. Knighton, B. Cottle, V. Dentan et al., "Development of an alveolar transbronchial catheter for concurrent fiber opticsbased imaging and fluid delivery," Journal of Medical Devices, vol. 12, no. 3, article 035003, 2018.

[31] W. C. Parks and S. D. Shapiro, "Matrix metalloproteinases in lung biology," Respiratory Research, vol. 2, no. 1, pp. 10-19, 2000.

[32] M. M. Gueders, J.-M. Foidart, A. Noel, and D. D. Cataldo, "Matrix metalloproteinases (MMPs) and tissue inhibitors of MMPs in the respiratory tract: potential implications in asthma and other lung diseases," European Journal of Pharmacology, vol. 533, no. 1-3, pp. 133-144, 2006.

[33] M. Toth and R. Fridman, "Assessment of gelatinases (MMP-2 and MMP-9 by gelatin zymography)," in Metastasis Research Protocols: Volume I: Analysis of Cells and Tissues, S. A. Brooks and U. Schumacher, Eds., pp. 163-174, Humana Press, Totowa, NJ, 2001.

[34] M. Salaün, J. Peng, H. H. Hensley et al., "MMP-13 in-vivo molecular imaging reveals early expression in lung adenocarcinoma," PLoS One, vol. 10, no. 7, article e0132960, 2015.

[35] B. Waschkau, A. Faust, M. Schäfers, and C. Bremer, "Performance of a new fluorescence-labeled MMP inhibitor to image tumor MMP activity in vivo in comparison to an MMPactivatable probe," Contrast Media \& Molecular Imaging, vol. 8, no. 1, pp. 1-11, 2013.
[36] J. O. McIntyre, R. L. Scherer, and L. M. Matrisian, "Near-infrared optical proteolytic beacons for in vivo imaging of matrix metalloproteinase activity," Methods in Molecular Biology (Clifton, N.J.), vol. 622, pp. 279-304, 2010.

[37] B.-W. Xie, I. M. Mol, S. Keereweer et al., "Dual-wavelength imaging of tumor progression by activatable and targeting near-infrared fluorescent probes in a bioluminescent breast cancer model," PLoS One, vol. 7, no. 2, article e31875, 2012.

[38] L. Zhu, Y. Ma, D. O. Kiesewetter et al., "Rational design of matrix metalloproteinase-13 activatable probes for enhanced specificity," ACS Chemical Biology, vol. 9, no. 2, pp. 510-516, 2013.

[39] T. Ma, Y. Hou, J. Zeng et al., "Dual-ratiometric target-triggered fluorescent probe for simultaneous quantitative visualization of tumor microenvironment protease activity and $\mathrm{pH}$ in vivo," Journal of the American Chemical Society, vol. 140, no. 1, pp. 211-218, 2017.

[40] P. A. Barber, D. Rushforth, S. Agrawal, and U. I. Tuor, "Infrared optical imaging of matrix metalloproteinases (MMPs) up regulation following ischemia reperfusion is ameliorated by hypothermia," BMC Neuroscience, vol. 13, no. 1, p. 76, 2012.

[41] P. B. Satkunananthan, M. J. Anderson, N. M. de Jesus, D. R. Haudenschild, C. M. Ripplinger, and B. A. Christiansen, "In vivo fluorescence reflectance imaging of protease activity in a mouse model of post-traumatic osteoarthritis," Osteoarthritis and Cartilage, vol. 22, no. 10, pp. 1461-1469, 2014.

[42] T. Fukui, E. Tenborg, J. H. N. Yik, and D. R. Haudenschild, "In-vitro and in-vivo imaging of MMP activity in cartilage and joint injury," Biochemical and Biophysical Research Communications, vol. 460, no. 3, pp. 741-746, 2015.

[43] H. Cho, F.-U.-R. Bhatti, T. W. Yoon, K. A. Hasty, J. M. Stuart, and A.-K. Yi, "Non-invasive dual fluorescence in vivo imaging for detection of macrophage infiltration and matrix metalloproteinase (MMP) activity in inflammatory arthritic joints," Biomedical Optics Express, vol. 7, no. 5, pp. 1842-1852, 2016.

[44] H. Järveläinen, A. Sainio, M. Koulu, T. N. Wight, and R. Penttinen, "Extracellular matrix molecules: potential targets in pharmacotherapy," Pharmacological Reviews, vol. 61, no. 2, pp. 198-223, 2009.

[45] R. Ramachandran, C. Altier, K. Oikonomopoulou, and M. D. Hollenberg, "Proteinases, their extracellular targets, and inflammatory signaling," Pharmacological Reviews, vol. 68, no. 4, pp. 1110-1142, 2016.

[46] N. B. Leighl, L. Paz-Ares, J. Y. Douillard et al., "Randomized phase III study of matrix metalloproteinase inhibitor BMS275291 in combination with paclitaxel and carboplatin in advanced non-small-cell lung cancer: National Cancer Institute of Canada-Clinical Trials Group Study BR.18," Journal of Clinical Oncology, vol. 23, no. 12, pp. 2831-2839, 2005.

[47] C. Haslett, L. A. Guthrie, M. M. Kopaniak, Johnston RB Jr, and P. M. Henson, "Modulation of multiple neutrophil functions by preparative methods or trace concentrations of bacterial lipopolysaccharide," The American Journal of Pathology, vol. 119, no. 1, pp. 101-110, 1985. 